\title{
A Comparison of Fast Kurtogram and SFLA based-ED Techniques for Bearing Fault Detection
}

\author{
Duc Hoang Nguyen \\ Faculty of Electrical and Electronics Engineering \\ HCMC University of Technology \\ Ho Chi Minh City, Vietnam
}

\begin{abstract}
The paper proposes a method using Shuffled Frog Leaping Algorithm (SFLA) to identify the optimal frequencies (center frequency and bandwith) of the bandpass filter. Addtion, fast kurtogram is also used to find the optimal bandpass filter. Simulated results on the data sets of the CWRU Bearing Data Center verify the effectiveness of SFLA approach, and show that the proposed method outperforms fast kurtogram.
\end{abstract}

\section{General Terms}

Optimization.

\section{Keywords}

Envelope detection, Fast kurtogram, SFLA, Bearing diagnosis.

\section{INTRODUCTION}

Rolling element bearings (REBs) are one of the most important elements of rotating machines. Their failures, such as outer race faults, inner race faults or rolling element faults, are the most frequent reasons for machine breakdown. Hence, early detecting these failures will prevent breakdown occurring. Vibration signals generated by faults in REBs have been widely studied and very powerful diagnostic techniques are now available [1].

Antoni [2] introduced the fast kurtogram which uses the kurtosis to determine the optimal bandpass filter. The vibration signal is band-pass filtered at various narrow-bands, and the kurtosis value determines the extent of impulsiveness in the filtered data. The filter specification returning the highest kurtosis can be selected as the optimal narrow-band for further envelope spectrum analysis.

SFLA is a swam-based optimization method and has been successfully applied to solve various optimization problems such as combinatorial optimization problems [3], powerquality improvement problems [4], application to $0 / 1$ knapsack problem [5], preemptive project scheduling problems with resource vacations based on patterson set [6], optimizing water distribution networks [7], economic dispatch with valve loading effect [8], designing fuzzy controller [9]. In this paper, SFLA is used to find optimal frequencies of the bandpass filter for bearing diagnosis.

The remaining paper is organized as follows: Section 2 presents review of the fast kurtogram and Shuffled Frog Leaping Algorithm. Section 3 introduces the fundamentals of the bearing fault detection as well as envelope detection technique. Section 4 shows the obtained results and Section 5 concludes this paper.

\section{REVIEW OF FAST KURTOGRAM AND SHUFFLED FROG LEAPING ALGORITHM}

\subsection{Fast kurtogram}

Based on the Wold-Cramer representation, a zero-mean nonstationary random process $x(n)$ can be decomposed into:

$$
x(n)=\int_{-1 / 2}^{+1 / 2} H(n, f) e^{j 2 \pi f t} d Z_{x}(f)
$$

where $d Z_{x}(f)$ is an orthonormal spectral increment and where $H(n, f)$ is the complex envelope of $x(n)$ at frequency $f$. The spectral kurtosis can then be defined as the fourth-order normalised cumulant:

$$
K_{x}(f)=\frac{\left\langle|H(n, f)|^{4}\right\rangle}{\left\langle|H(n, f)|^{2}\right\rangle^{2}}-2
$$

where $\langle f(n)\rangle=\lim _{N \rightarrow \infty} N^{-1} \sum_{N} f(n)$ stands for the temporal average of function $f(n)$ and the constant -2 comes from the fact that $H(n, f)$ is complex. [2]

The kurtogram is a fourth-order spectral analysis tool for detecting and characterising non-stationarities in a signal. It relies on the assertion that each type of transient is associated with an optimal (frequency/frequency resolution) dyad $(f ; \Delta f)$ which maximises the kurtosis. [2]

Computation of the kurtogram for all possible combinations of centre frequencies $(f)$ and bandwidths $(\Delta f)$ is obviously costly and not convenient for practical purposes. Antoni [2] developed a fast method based on $1 / 3$ binary filter banks to calculated the spectral kurtosis. The paving of $1 / 3$ binary filter banks is shown in Fig. 1.

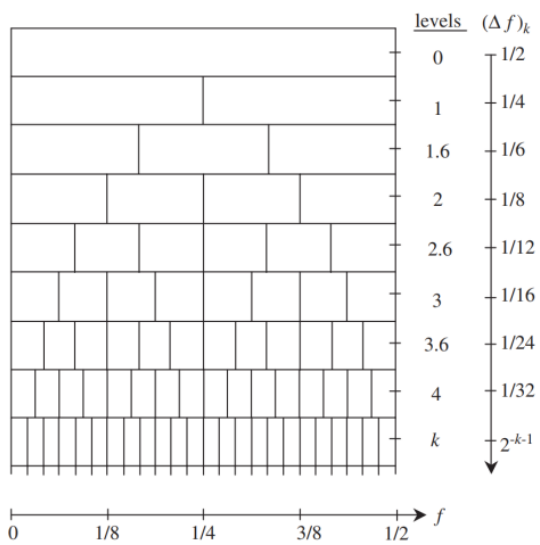

Fig. 1: Paving of the original kurtogram proposed by Antoni [2] 


\subsection{Shuffled frog leaping algorithm}

The SFLA is a meta-heuristic optimization method that mimics the memetic evolution of a group of frogs when seeking for the location that has the maximum amount of available food.

The algorithm contains the elements of the local search and global information exchange. The SFLA involves a population of the possible solutions defined by a set of virtual frogs that is partitioned into subsets referred to as memeplexes. Within each memeplex, the individual frog holds ideas that can be influenced by the ideas of other frogs, and the ideas can evolve through a process of the memetic evolution. The SFLA performs simultaneously an independent local search in each memeplex using a particle swarm optimization-like method. To ensure global exploration, after a defined number of memeplex evolution steps (i.e. local search iterations), the virtual frogs are shuffled and reorganized into new memeplexes in a technique similar to that used in the shuffled complex evolution algorithm.

In addition, to provide the opportunity for random generation of the improved information, random virtual frogs are generated and substituted in the population if the local search cannot find better solutions. The local searches and the shuffling processes continue until defined convergence criteria are satisfied. The flowchart of the SFLA is illustrated in Fig 2.

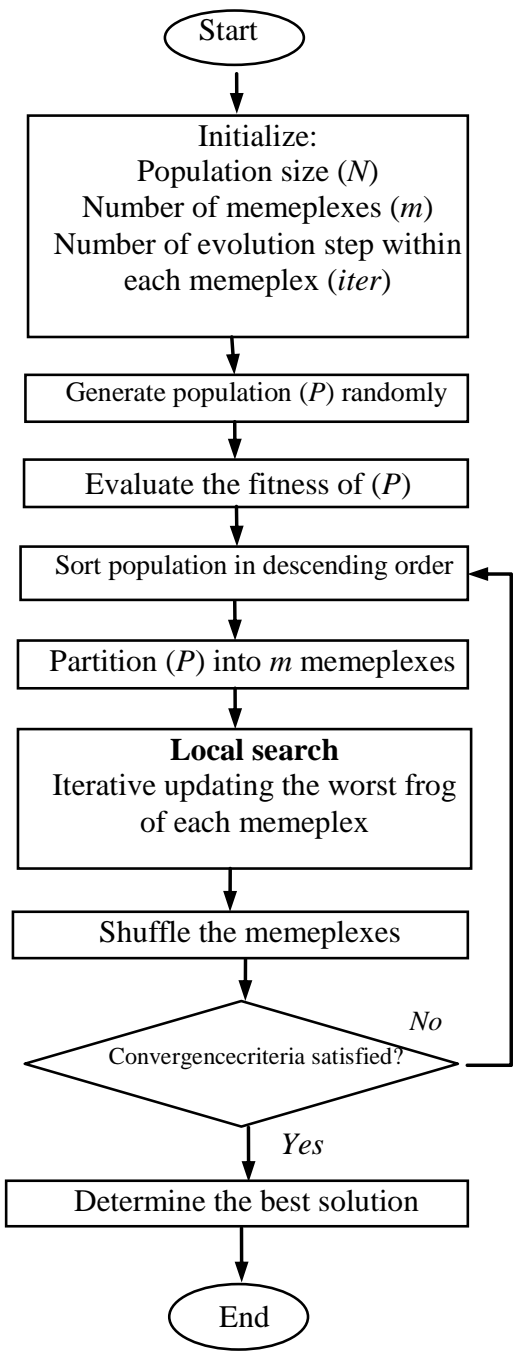

Fig. 2: Flowchart of the SFLA
The idea updating frog leaping rule which is expressed as:

$$
\begin{gathered}
D=\operatorname{r.c}\left(X_{b}-X_{w}\right) \\
X_{w}(\text { new })=X_{w}+D,\|D\| \leq D_{\max }
\end{gathered}
$$

Where $X_{b}$ and $X_{w}$ are identified as the frogs with the best and the worst fitness, respectively; $r$ is a random number between 0 and $1 ; c$ is a constant chosen in the range between 1 and 2 . Refer to [8] and [9] for more information.

\section{BEARING FAULT DETECTION}

\subsection{Fundamentals}

Fig. 3 shows typical acceleration signals produced by localised faults in the various components of a rolling element bearing, and the corresponding envelope signals produced by amplitude demodulation.[1]

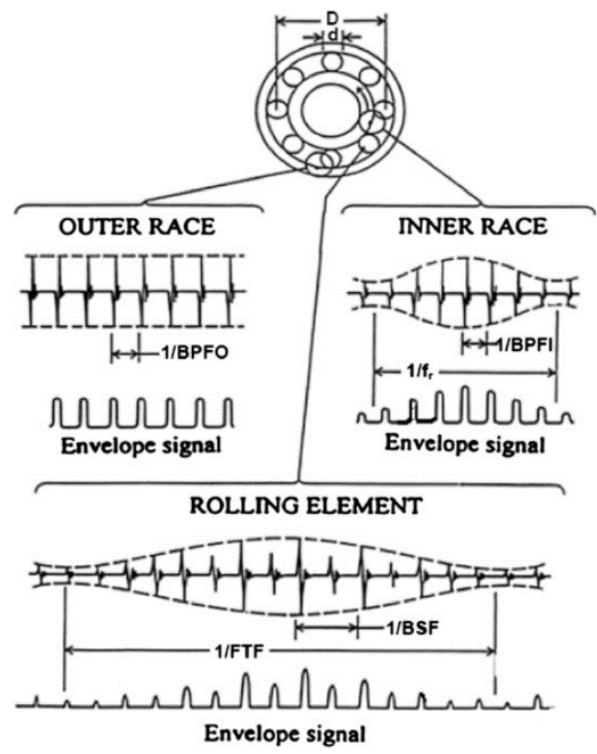

Fig. 3: Typical signals and envelope signals from local faults in rolling element bearings [1]

The defect frequencies of bearing are calculated from geometric dimensions of the bearing, number of balls and shaft frequency as given as follows:

Ball Pass Frequency Outer Race:

$$
B P F O=\frac{n f_{r}}{2}\left\{1-\frac{d}{D} \cos \emptyset\right\}
$$

Ball Pass Frequency Inner Race:

$$
B P F I=\frac{n f_{r}}{2}\left\{1+\frac{d}{D} \cos \emptyset\right\}
$$

Where $f_{r}$ is the shaft speed, $n$ is the number of rolling elements, and $\emptyset$ is the angle of the load from the radial plane.

\subsection{Envelope detection technique}

As shown in [1], the spectrum of the raw signal contains little useful information in order to be able to detect bearing faults, and over many years the envelope analysis has been established as the benchmark method for bearing diagnostics, where a signal is bandpass filtered in a high frequency band in which the fault impulses are amplified by structural resonances. It is then amplitude demodulated to form the envelope signal, whose spectrum contains the desired diagnostic information. 


\subsection{Procedure to detect bearing fault}

Fault identification process using SFLA or fast kurtogram is shown in Fig. 4. SFLA is used to maximise amplitude of the envelope spectrum:

$$
A=f\left(X, F_{c f}, B W, \text { freq }, F_{s}\right)
$$

Where, $A$ is the amplitude of the envelope spectrum at defect frequency of the bearin; $F_{c f}$ is the center frequency and $B W$ is the bandwith of the bandpass filter; freq is the defect frequency of the bearing (BPFO, BPFI); $F_{S}$ is the sample frequency.

In this paper, SFLA is used to maximise $A$. The central frequency and bandwidth of bandpass filter were optimised by SFLA so as to achieve highest $A$ at the desired defect frequency in envelope spectrum.

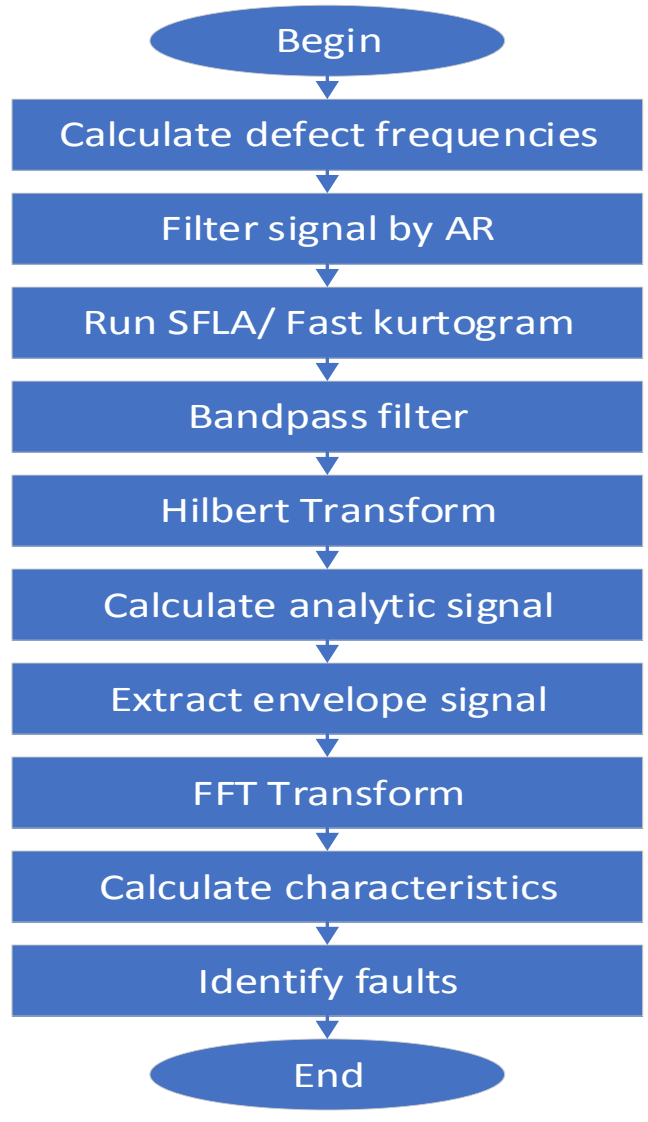

Fig. 4: Fault identification process

\section{EXPERIMENTAL RESULTS}

\subsection{Data sets}

Vibration signals used in this paper were provided by the bearing test rig of the CWRU bearing data center. This experimental setup is shown in Fig. 5, which consists of a two horsepower Reliance Electric motor, a torque transducer/encoder, a dynamometer and control electronics. Further details can be found on the CWRU Bearing Data Center Website.

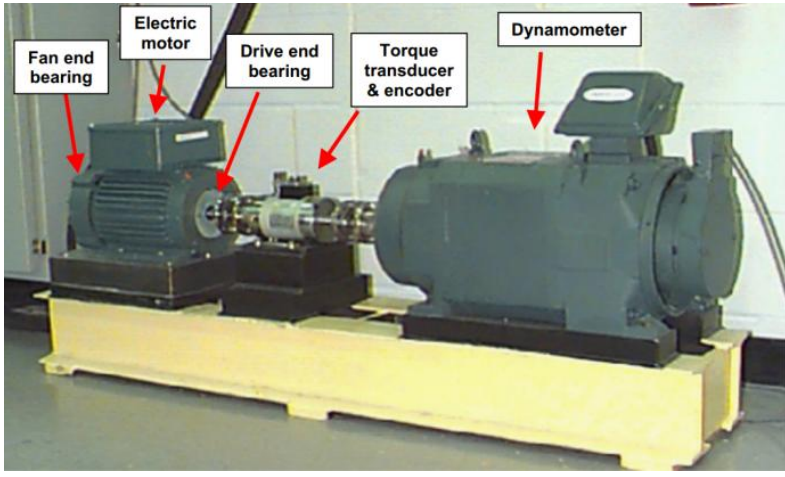

Fig. 5: The bearing test rig

Data sets provided by CWRU give many cases that is combination of the bearing fault types such as, outer race fault, inner race fault, rolling element fault, fault size and motor load. In this paper, the following combinations are considered as Table 1 and 2 .

Table 1. Outer race fault case

\begin{tabular}{|c|c|c|c|}
\hline Case & Fault size & Motor load & Speed \\
\hline 1 & $0.18 \mathrm{~mm}$ & $0 \mathrm{hp}$ & $1796 \mathrm{RPM}$ \\
\hline 2 & $0.53 \mathrm{~mm}$ & $3 \mathrm{hp}$ & $1719 \mathrm{RPM}$ \\
\hline
\end{tabular}

Table 2. Inner race fault case

\begin{tabular}{|c|c|c|c|}
\hline Case & Fault size & Motor load & Speed \\
\hline 3 & $0.18 \mathrm{~mm}$ & $0 \mathrm{hp}$ & 1796 RPM \\
\hline 4 & $0.53 \mathrm{~mm}$ & $3 \mathrm{hp}$ & 1727 RPM \\
\hline
\end{tabular}

Fig. 6 shows vibration signal of case 1 .

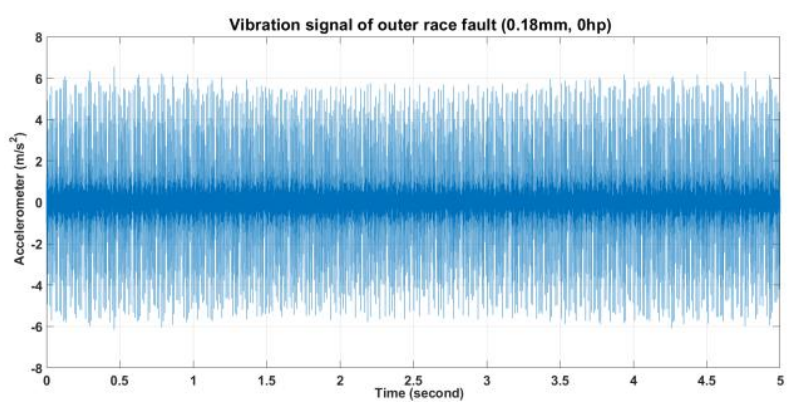

Fig. 6: Vibration signal of case 1

The next sections present the results of identifying the fault of the bearing.

\subsection{Detect fault using fast kurtogram}

4.2.1 Case 1: fault size 0.18mm, motor load 0hp In this case, defect frequencies are given as Table 3 (using equations 5,6 )

Table 3. The defect frequencies of the case 1

\begin{tabular}{|c|c|}
\hline BPFO $(\mathrm{Hz})$ & BPFI $(\mathrm{Hz})$ \\
\hline 107.3 & 162.1 \\
\hline
\end{tabular}


Fig. 7 is the kurtogram of the vibration signal after filtered by AR. The region has largest kurtosis (yellow color) is resonance region. The parameters of the bandpass filter obtained as follows: central frequency is $5625 \mathrm{~Hz}\left(f_{c}=\right.$ $5625 \mathrm{~Hz})$, bandwidth is $750 \mathrm{~Hz}(B W=750 \mathrm{~Hz})$. Then, this filter is used to find envolope signal.

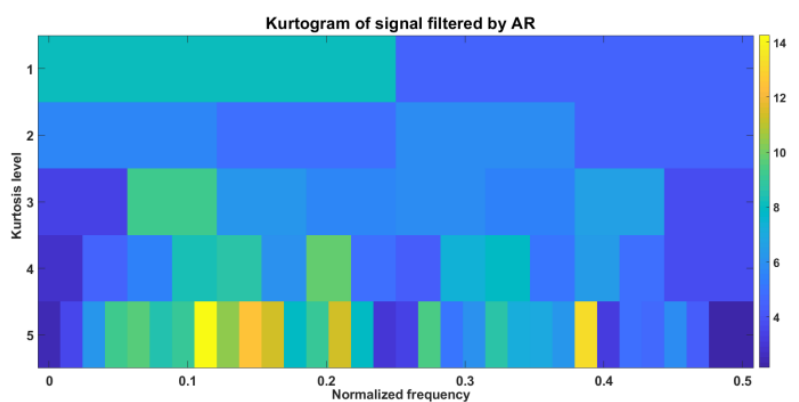

Fig. 7: Kurtogram of vibration signal

Fig. 8 is the spectrum of the envolope signal. From figure, it can be seen that peak of the spectrum at frequency $107.7 \mathrm{~Hz}$. This value is very much larger than others. This frequency is the defect frequency of the outer race fault.

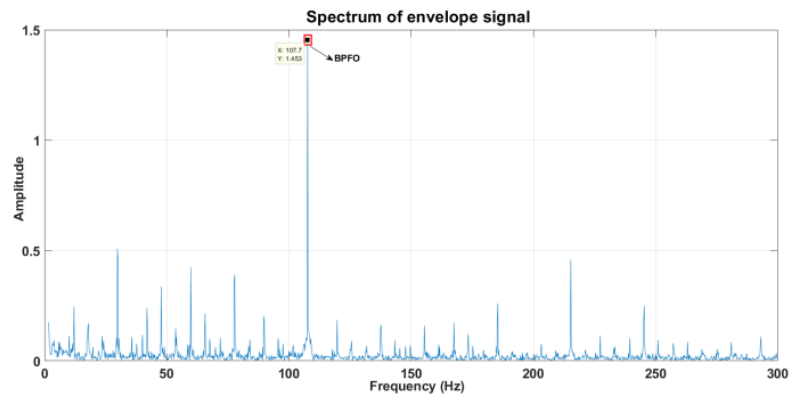

Fig. 8: Spectrum of the envolope signal

After spectrum of envelope is extracted, its peaks at defect frequencies are checked for how much amplification and Peak to Mean Ratio (PMR) has been attained [12]. Results are shown in Table 4.

Table 4. The characteristic values of the case 1

\begin{tabular}{|c|c|c|}
\hline Defect frequencies (Hz) & Amplification & PMR \\
\hline 107.85 & 4.47 & 2052.19 \\
\hline 161.50 & 0.03 & 106.90 \\
\hline
\end{tabular}

Remark: Based on Table 4, it can be seen that at defect frequency $107.85 \mathrm{~Hz}$, amplification and PMR values are very much larger than values at other frequencies. That verifies the bearing is outer race fault, which is compatible with test data.

\subsubsection{Case 2: fault size $0.53 \mathrm{~mm}$, motor load $3 \mathrm{hp}$} In this case, defect frequencies are given as Table 5.

Table 5. The defect frequencies of the case 2

\begin{tabular}{|c|c|}
\hline BPFO $(\mathrm{Hz})$ & BPFI $(\mathrm{Hz})$ \\
\hline 102.7 & 155.15 \\
\hline
\end{tabular}

Fig. 9 is the kurtogram of the vibration signal after filtered by AR. The region has largest kurtosis (yellow color) is resonance region. The parameters of the bandpass filter obtained as follows: $f_{c}=18000 \mathrm{~Hz}, \mathrm{BW}=12000 \mathrm{~Hz}$.

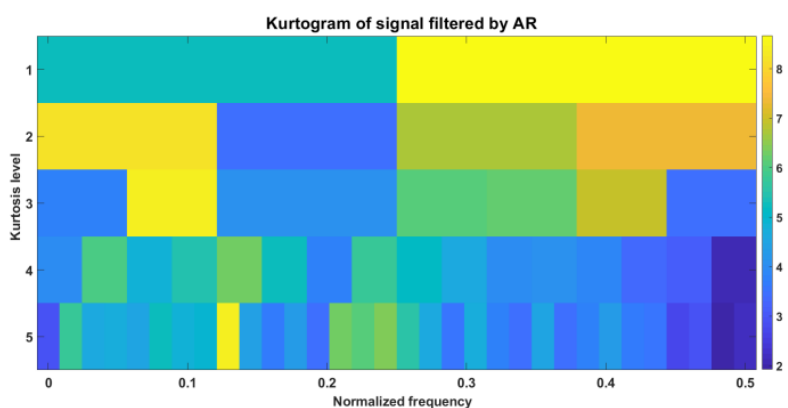

Fig. 9: Kurtogram of vibration signal

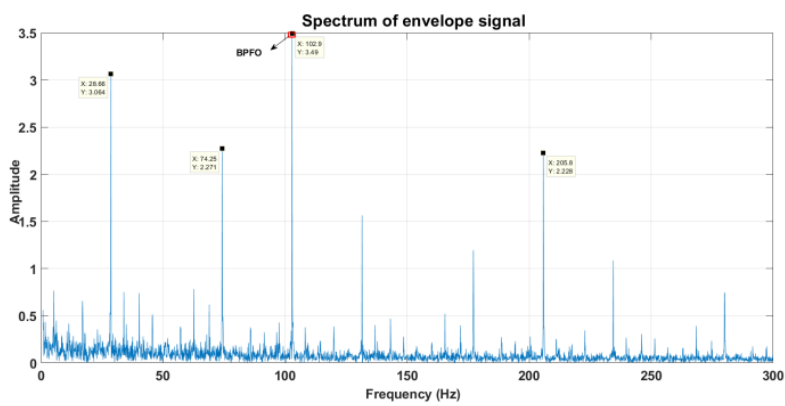

Fig. 10: Spectrum of the envolope signal

Amplification and PMR values are shown in Table 6.

Table 6. The characteristic values of the case 2

\begin{tabular}{|c|c|c|}
\hline Defect frequencies (Hz) & Amplification & PMR \\
\hline 103.00 & 24.23 & 385.94 \\
\hline 153.99 & 0.10 & 19.73 \\
\hline
\end{tabular}

Remark: At defect frequency $103.00 \mathrm{~Hz}$, amplification and PMR values are very much larger than values at other frequencies as case 1 .

4.2.3 Case 3: fault size 0.18mm, motor load 0hp In this case, defect frequencies are given as Table 7.

Table 7. The defect frequencies of the case 3

\begin{tabular}{|c|c|}
\hline BPFO $(\mathrm{Hz})$ & BPFI $(\mathrm{Hz})$ \\
\hline 107.3 & 162.1 \\
\hline
\end{tabular}

Fig. 11 is the kurtogram of the vibration signal after filtered by AR. The region has largest kurtosis (yellow color) is resonance region. The parameters of the bandpass filter obtained as follows: $f_{c}=2625 \mathrm{~Hz}, B W=750 \mathrm{~Hz}$.

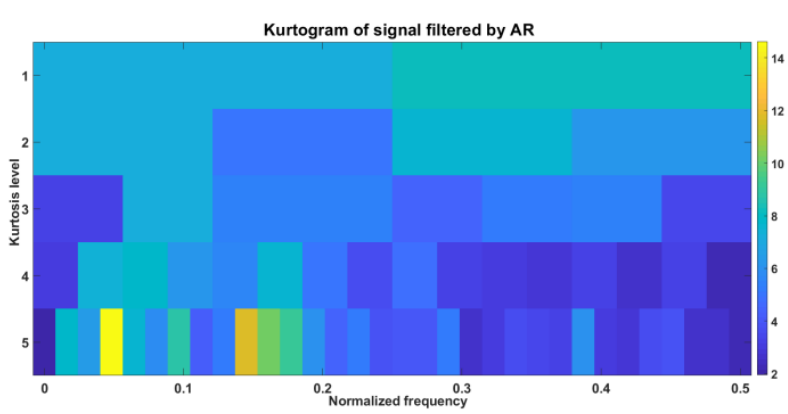

Fig. 11: Kurtogram of vibration signal 


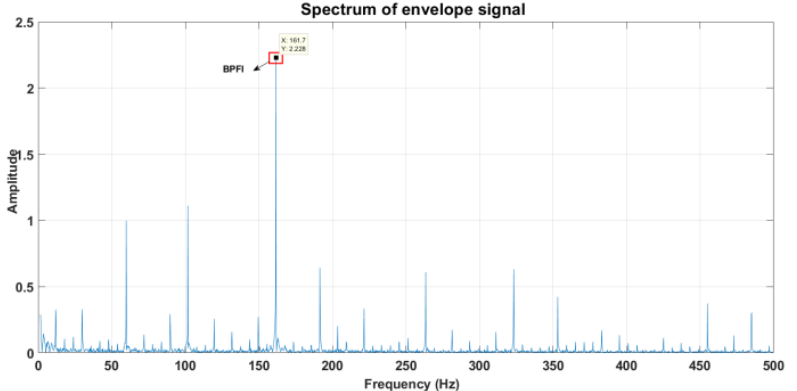

Fig. 12: Spectrum of the envolope signal

Amplification and PMR values are shown in Table 8.

Table 8. The characteristic values of the case 3

\begin{tabular}{|c|c|c|}
\hline Defect frequencies (Hz) & Amplification & PMR \\
\hline 108.03 & 0.41 & 57.02 \\
\hline 161.86 & 0.68 & 2728.76 \\
\hline
\end{tabular}

Remark: At defect frequency $161.86 \mathrm{~Hz}$, PMR values are very much larger than value at other frequencies.

\subsubsection{Case 4: fault size 0.53mm, motor load 3hp} In this case, defect frequencies are given as Table 9.

Table 9. The defect frequencies of the case 4

\begin{tabular}{|c|c|}
\hline BPFO $(\mathrm{Hz})$ & BPFI $(\mathrm{Hz})$ \\
\hline 107.3 & 162.1 \\
\hline
\end{tabular}

Fig. 13 is the kurtogram of the vibration signal after filtered by AR. The region has largest kurtosis (yellow color) is resonance region. The parameters of the bandpass filter obtained as follows: $f_{c}=2625 \mathrm{~Hz}, \mathrm{BW}=750 \mathrm{~Hz}$.

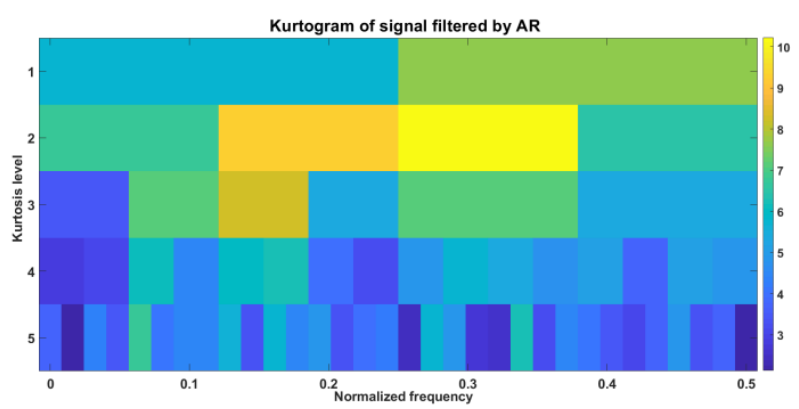

Fig. 13: Kurtogram of vibration signal

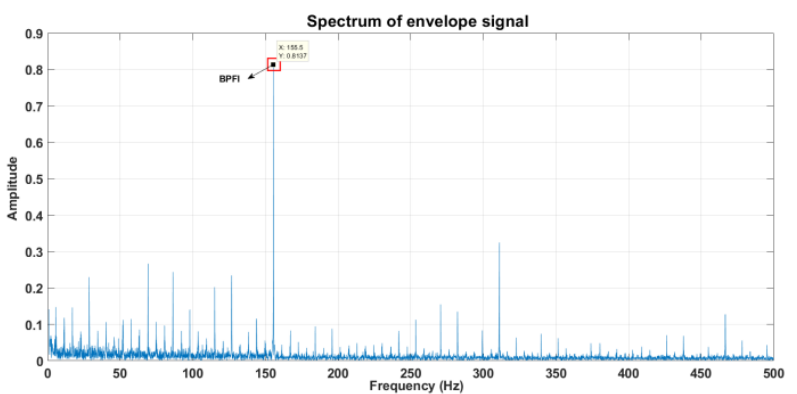

Fig. 14: Spectrum of the envolope signal
Amplification and PMR values are shown in Table 10.

Table 10. The characteristic values of the case 4

\begin{tabular}{|c|c|c|}
\hline Defect frequencies (Hz) & Amplification & PMR \\
\hline 103.82 & 4.24 & 57.09 \\
\hline 155.64 & 1.56 & 575.52 \\
\hline
\end{tabular}

Remark: At defect frequency $155.64 \mathrm{~Hz}$, PMR values are very much larger than value at other frequencies.

\subsection{Detect fault using SFLA}

4.3.1 Case 1: fault size 0.18mm, motor load 0hp In this case, SFLA is used to find parameters of the bandpass filter. Results obtained after running SFLA at defect frequency of outer race fault are: $f_{c}=6921 \mathrm{~Hz}, B W=$ $10786 \mathrm{~Hz}$.

Fig. 15 is the spectrum of the envelope signal after bandpass filtered.

Amplification and PMR values are shown in Table 11.

Table 11. The characteristic values of the case 1

\begin{tabular}{|c|c|c|}
\hline Defect frequencies (Hz) & Amplification & PMR \\
\hline 107.85 & 18.84 & 738.15 \\
\hline 161.50 & 0.03 & 7.75 \\
\hline
\end{tabular}

4.3.2 Case 2: fault size $0.53 \mathrm{~mm}$, motor load $3 \mathrm{hp}$ Similarly, results obtained after running SFLA at defect frequency of outer race fault are: $f_{c}=32121 \mathrm{~Hz}, B W=$ $37086 \mathrm{~Hz}$.

Fig. 16 is the spectrum of the envelope signal after bandpass filtered.

Amplification and PMR values are shown in Table 12.

Table 12. The characteristic values of the case 2

\begin{tabular}{|c|c|c|}
\hline Defect frequencies (Hz) & Amplification & PMR \\
\hline 103.00 & 32.80 & 253.49 \\
\hline 153.99 & 0.13 & 12.08 \\
\hline
\end{tabular}

\subsubsection{Case 3: fault size $0.18 \mathrm{~mm}$, motor load $0 \mathrm{hp}$} Similarly, results obtained after running SFLA at defect frequency of outer race fault are: $f_{c}=6635 \mathrm{~Hz}, B W=$ $10296 \mathrm{~Hz}$.

Fig. 17 is the spectrum of the envelope signal after bandpass filtered.

Amplification and PMR values are shown in Table 13.

Table 13. The characteristic values of the case 3

\begin{tabular}{|c|c|c|}
\hline Defect frequencies (Hz) & Amplification & PMR \\
\hline 107.85 & 1.34 & 13.11 \\
\hline 161.86 & 2.10 & 593.73 \\
\hline
\end{tabular}


4.3.4 Case 4: fault size $0.53 \mathrm{~mm}$, motor load $3 \mathrm{hp}$ Similarly, results obtained after running SFLA at defect frequency of outer race fault are: $f_{c}=20618.5 \mathrm{~Hz}, B W=$ $38539 \mathrm{~Hz}$.

Fig. 18 is the spectrum of the envelope signal after bandpass filtered.

Amplification and PMR values are shown in Table 14.

Table 14. The characteristic values of the case 4

\begin{tabular}{|c|c|c|}
\hline Defect frequencies (Hz) & Amplification & PMR \\
\hline 103.82 & 7.77 & 21.38 \\
\hline 155.64 & 4.17 & 314.28 \\
\hline
\end{tabular}

Remark: Similar to fast kurtogram, SFLA gives values of amplification and PMR at defect frequency very much larger than value at other frequencies.

\subsection{Comparison}

This section compares the obtained results when using fast kurtogram and SFLA.

\subsubsection{Outer race fault case}

Fig. $x$ and Fig.x compare the spectrum of the envelope when using fast kurtogram and SFLA for two cases 1 and 2 .

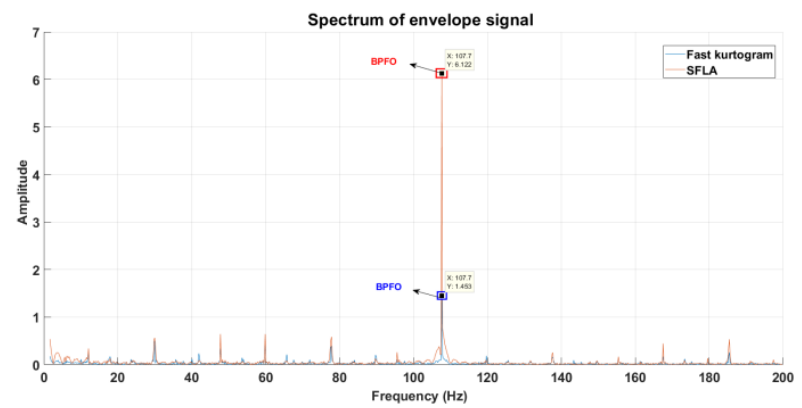

Fig. 15: Spectrum of the envolope signal in case 1

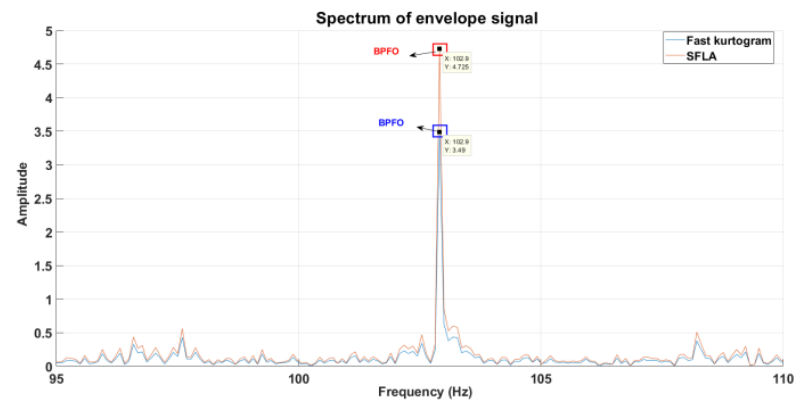

Fig. 16: Spectrum of the envolope signal in case 2

\subsubsection{Inner race fault case}

Fig. $x$ and Fig. $x$ compare the spectrum of the envelope when using fast kurtogram and SFLA for two cases 3 and 4.

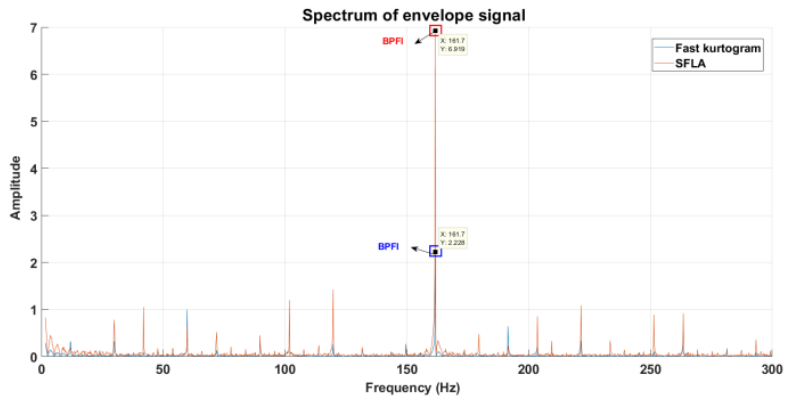

Fig. 17: Spectrum of the envolope signal in case 3

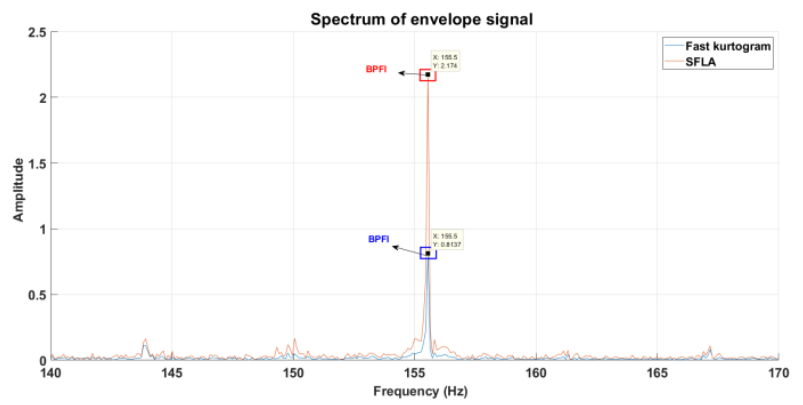

Fig. 18: Spectrum of the envolope signal in case 4

Remark: In all cases, SFLA gives results better than fast kurtogram. Amplitude of spectrum of the envelope signal as using SFLA has value larger than value as using fast kurtogram, especially in cases 1 and 3 . This means that, SFLA gives fault diagnosis results more exact than fast kurtogram.

\section{CONCLUSION}

In this paper, SFLA is applied to find optimally the frequencies of the bandpass filter for bearing diagnosis. The simulated results on data sets of Case Western Reserve University Bearing Data Center show that SFLA outperforms the fast kurtogram about identifying the defect frequencies of the bearing. In the future, the author will continue extending these results by comparing the SFLA with other swarm intelligence optimisation techniques such as $\mathrm{ACO}, \mathrm{PSO}, \ldots$

\section{REFERENCES}

[1] R. B. Randall and J. Antoni. 2011. Rolling element bearing diagnostics - A tutorial. Mechanical Systems and Signal Processing. Elsevier Ltd.

[2] J. Antoni. 2007. Fast computation of the kurtogram for the detection of transient faults. Mechanical Systems and Signal Processing. Elsevier Ltd.

[3] Muzaffar Eusuff, Kevin Lansey and Fayzul Pasha. 2006. Shuffled frog-leaping algorithm: a memetic metaheuristic for discrete optimization. Engineering Optimization. Taylor \& Francis Online.

[4] A. Darvishi, A. Alimardani, B. Vahidi, S.H. Hosseinian. 2014. Shuffled Frog-Leaping Algorithm for Control of Selective and Total Harmonic Distortion. Journal of Applied Research and Technology.

[5] Kaushik Kumar Bhattacharjee, S.P. Sarmah. 2014. Shuffled frog leaping algorithm and its application to 0/1 knapsack problem. Applied Soft Computing.

[6] Yi Han, et al. 2013. Shuffled Frog Leaping Algorithm for Preemptive Project Scheduling Problems with Resource Vacations Based on Patterson Set. Journal of Applied Mathematics.

[7] Daniel Mora-Melia, Pedro L. Iglesias-Rey, F. Javier 
Martínez-Solano and Pedro Muñoz-Velasco. 2016. The Efficiency of Setting Parameters in a Modified Shuffled Frog Leaping Algorithm Applied to Optimizing Water Distribution Networks. Water, MDPI.

[8] Dina M. Said, Nabil M. Hamed, Almoataz Y. Abdelaziz. 2016. Shuffled Frog Leaping Algorithm for Economic Dispatch with Valve Loading Effect. International Electrical Engineering Journal.

[9] Duc-Hoang Nguyen and Thai-Hoang Huynh. 2008. A SFLA-Based Fuzzy Controller for Balancing a Ball and Beam System. Tenth IEEE International Conference on Control, Automation, Robotics and Vision (ICARCV
2008).

[10] Loparo KA. Case Western Reserve University Bearing Data Center. http://csegroups.case.edu/bearingdatacenter/

[11] Wade A. Smith, Robert B. Randall. 2015. Rolling element bearing diagnostics using the Case Western Reserve University data: A benchmark study. Mechanical Systems and Signal Processing. Elsevier Ltd.

[12] S. Tyagi, S.K. Panigrahi. 2017. An improved envelope detection method using Particle Swarm Optimisation for rolling element bearing fault diagnosis. Journal of Computational Design and Engineering. 